\title{
Retreatment With Anti-PD-L1 Antibody in Advanced Non-small Cell Lung Cancer Previously Treated With Anti-PD-1 Antibodies
}

\author{
KOHEI FUJITA ${ }^{1}$, NAOHIRO UCHIDA ${ }^{1}$, YUKI YAMAMOTO ${ }^{1,2}$, OSAMU KANAI ${ }^{1}$, \\ MISATO OKAMURA ${ }^{1}$, KOICHI NAKATANI $^{1}$, SATORU SAWAI ${ }^{3}$ and TADASHI MIO ${ }^{1}$ \\ ${ }^{1}$ Division of Respiratory Medicine, Center for Respiratory Diseases, \\ National Hospital Organization Kyoto Medical Center, Kyoto, Japan; \\ ${ }^{2}$ Department of Drug Discovery for Lung Diseases, Graduate School of Medicine, Kyoto University, Kyoto, Japan; \\ ${ }^{3}$ Division of Thoracic Surgery, Center for Respiratory Diseases, \\ National Hospital Organization Kyoto Medical Center, Kyoto, Japan
}

\begin{abstract}
Aim: To evaluate the efficacy and safety of retreatment with anti-programmed death (PD)-L1 antibody (atezolizumab) after anti-PD-1 antibody (nivolumab/ pembrolizumab) treatment in advanced non-small cell lung cancer (NSCLC) patients. Patients and Methods: We retrospectively reviewed 18 NSCLC patients who received atezolizumab after anti-PD-1 antibody treatment. Data on patient characteristics, number of cycles of anti-PD-1 antibody and atezolizumab, regimens between anti-PD-1 antibody and atezolizumab, best response, and immune-related adverse events (irAEs) were collected and analyzed. Results: Nine patients a had high ( $\geq 50 \%)$ PD-L1 expression. The median number of cycles of atezolizumab was 3 (range=2-7). The median progression-free survival was $2.9 \pm 1.8$ months. Seven (38.9\%) and 11 (61.1\%) patients had stable and progressive disease, respectively. No patient achieved partial or complete response. There were no significant differences in the occurrence of irAEs between anti-PD-1 antibodies and atezolizumab. Conclusion: Preliminary results showed that patients previously treated with anti $P D-1$ antibodies received only limited benefit from subsequent atezolizumab.
\end{abstract}

Cancer immunotherapy has recently received remarkable attention, and cumulative evidence has established it as the

Correspondence to: Kohei Fujita, MD, Ph.D., Division of Respiratory Medicine, Center for Respiratory Diseases, National Hospital Organization Kyoto Medical Center, 1-1, Fukakusa, Mukaihata-Cho, Fushimi-Ku, Kyoto, Japan. Tel: +81 756419161, e-mail:kfujita-oka@umin.ac.jp

Key Words: Atezolizumab, immune checkpoint inhibitors, anti-PD-1 antibodies, lung cancer. fourth pillar of cancer therapy. The CheckMate and Keynote studies showed the superiority of anti-programmed death (PD)-1 antibodies over conventional cytotoxic chemotherapy as second-line therapy (1-3). As a result of these clinical trials, the anti-PD-1 antibodies nivolumab and pembrolizumab were approved for non-small cell lung cancer (NSCLC) patients. More recently, the OAK and PACIFIC studies also showed the benefits of anti-PD ligand 1 (PD-L1) antibodies for NSCLC patients $(4,5)$. The anti-PD-L1 antibodies atezolizumab and durvalumab have since been approved for second- or later-line therapy and maintenance therapy after chemoradiotherapy, respectively.

One of the main drawbacks of immunotherapy using immune checkpoint inhibitors (ICIs) is that only select patients are benefited. In the clinical setting, physicians often have to administer another chemotherapy after treatment failure of initial ICIs (6). However, there are few reports assessing the clinical benefits of subsequent use of similar ICIs (7-10). Although the use anti-PD-L1 antibody after antiPD-1 antibody treatment has become a growing concern in the clinical setting, there is no report addressing this issue. Therefore, in this study, we aimed to evaluate the efficacy and safety of re-treatment with atezolizumab after anti-PD1 antibody treatment.

\section{Patients and Methods}

This was a retrospective cohort study in the National Hospital Organization Kyoto Medical Center (Kyoto, Japan). Patients who received atezolizumab between January 2018 and December 2018 were reviewed. The observational period for evaluation of efficacy and safety was January 2018 to February 2019. At the time of this study, nivolumab and pembrolizumab were administered as anti-PD-1 antibody treatments, while atezolizumab and durvalumab as antiPD-L1 antibody treatments. Because durvalumab is approved for 
maintenance treatment after conventional chemoradiotherapy, patients who received durvalumab were excluded, thus atezolizumab was the only anti-PD-L1 antibody included in the study analysis. We reviewed all NSCLC patients who received atezolizumab who fulfilled the following criteria: (A) pathologically confirmed NSCLC, and (B) retreatment with atezolizumab after treatment with nivolumab and/or pembrolizumab. The following data were collected: patient characteristics, number of treatment cycles and progression-free survival (PFS) in patients treated with anti-PD-1 antibodies and atezolizumab, regimens between anti-PD-1 antibodies and atezolizumab, best response, and immune-related adverse events (irAEs).

Treatment response was evaluated according to the Response Evaluation Criteria in Solid Tumors version 1.1, and irAEs were evaluated according to the Common Terminology Criteria for Adverse Events version 4.0. This study was approved by our institutional review board.

\section{Results}

Patient characteristics. Between January 2018 and December 2018, 52 NSCLC patients were treated with atezolizumab. Out of these patients, 18 (34.6\%) had been previously treated with anti-PD-1 antibodies; 8 patients received nivolumab, 7 patients received pembrolizumab, and 3 patients received both nivolumab and pembrolizumab (Figure 1). We included these 18 patients for further analysis. The characteristics of the patients are shown in Table I. The mean age at induction of the initial anti-PD-1 antibody was $71.0 \pm 6.8$ years, and 14 patients had a history of smoking. Eight had adenocarcinoma histopathology and 9 had high $(\geq 50 \%)$ tumor PD-L1 expression at the time of diagnosis.

Results of initial treatment with anti-PD-1 antibodies (nivolumab/pembrolizumab). Although patients receiving nivolumab had low tumor PD-L1 expression, patients receiving pembrolizumab had a high tumor PD-L1 expression. All patients receiving nivolumab had previously received cytotoxic chemotherapy. The PFS was longer in patients receiving nivolumab than in patients receiving pembrolizumab (7.7 \pm 6.6 months $v s .5 .6 \pm 4.7$ months). More than half of the patients receiving initial treatment with antiPD-1 antibodies achieved disease control (stable disease $(\mathrm{SD})+$ partial response $(\mathrm{PR}))$ (Table II).

Results of subsequent treatment with anti-PD-L1 antibody (atezolizumab). Regarding the subsequent treatment with atezolizumab, the median number of cycles of atezolizumab was 3 (range=2-7). Nine of 18 patients had tumor PD-L1 expression. Seven of 18 patients achieved SD. Eleven of 18 patients showed progressive disease and received no benefits from atezolizumab. No patient achieved PR/CR. The PFS was only $2.9 \pm 1.8$ months, and the disease control rate was $38.9 \%$. Nine of 18 patients received cytotoxic chemotherapy between anti PD-1 antibodies and atezolizumab treatment.
Table I. Demographics and clinicopathological characteristics of patients $(n=18)$.

\begin{tabular}{lc}
\hline Characteristic & $\mathrm{n}(\%)$ \\
\hline Age, years & $71.0 \pm 6.8^{*}$ \\
Gender (female) & $7(38.9)$ \\
Smoking history & $14(77.8)$ \\
Body mass index, mg/m² & $21.3 \pm 3.3^{*}$ \\
Histopathology & \\
$\quad$ Adenocarcinoma & $8(44.4)$ \\
Squamous carcinoma & $6(33.3)$ \\
Adenosquamous carcinoma & $1(5.6)$ \\
NOS & $2(11.1)$ \\
Pleomorphic carcinoma & $1(5.6)$ \\
Performance status $(\geq 2)$ & $7(38.9)$ \\
Driver mutations & $0(0)$ \\
PD-L1 expression & \\
TPS $\geq 50 \%$ & $9(50.0)$ \\
$1 \% \leq$ TPS $<50 \%$ & $3(16.7)$ \\
TPS <1\% & $3(16.7)$ \\
NE & $3(16.7)$ \\
Clinical stage & \\
$3 A, 3 B$ & $9(50.0)$ \\
4 & $9(50.0)$ \\
Number of prior regimens before anti-PD-1 antibody** & $1(0-3)$ \\
\hline
\end{tabular}

*Data shown as mean \pm standard deviation. **Data shown as median (range). NOS, Not otherwise specified; PD-L1, programmed death ligand 1; TPS, tumor proportion score; NE, not evaluated; PD-1, programmed death-1.

The most frequently administered regimen was combination docetaxel and ramucirumab (Table III).

Profiles of immune-related adverse events. The occurrences of irAEs during both the initial anti-PD-1 antibody treatment and subsequent atezolizumab are shown in Table IV. Skin rash and fever were more frequently observed during atezolizumab treatment than during treatment with anti-PD-1 antibodies. Diarrhea was observed only during treatment with anti-PD-1 antibodies, and mental disorder was observed only during treatment with atezolizumab. Two patients had severe bacterial pneumonia during atezolizumab treatment.

\section{Discussion}

Previous clinical studies have not clarified whether retreatment with anti-PD-1/PD-L1 antibodies provides benefits. Several studies had only limited data regarding retreatment with ICIs. Two studies in melanoma patients suggested that response to the first anti-PD-1 antibody was predictive of the efficacy of retreatment with a second antiPD-1 antibody $(7,8)$. Two retrospective studies in lung cancer patients also showed the efficacy of retreatment. One study showed that patients with very high $(\geq 80 \%)$ tumor PD-L1 expression received benefits from retreatment (9). The other 


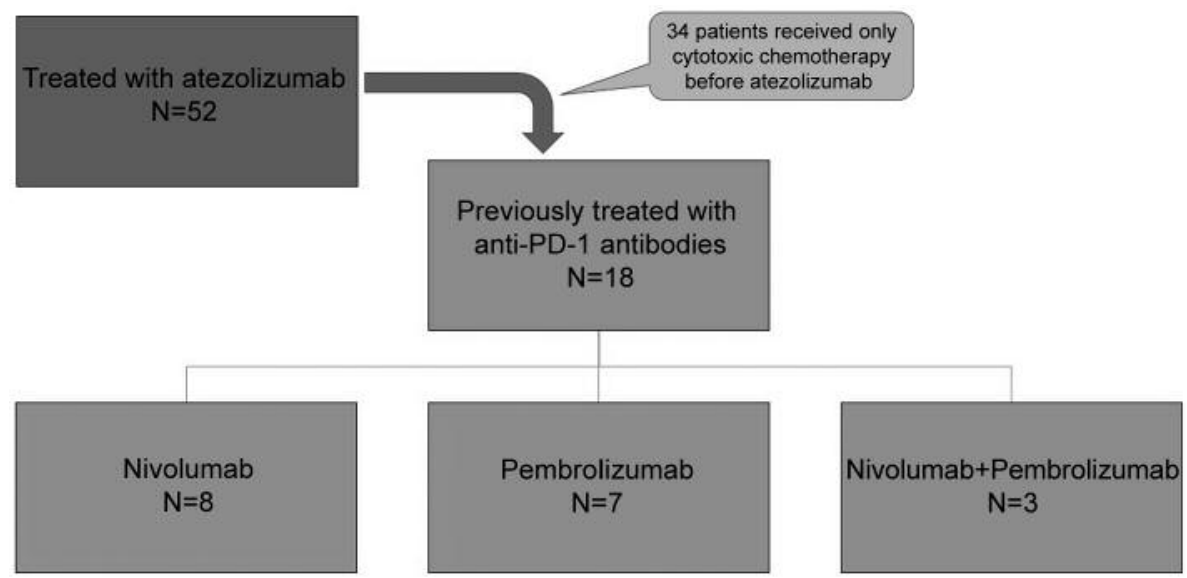

Figure 1. Flow chart of the study population.

Table II. Data about patients that received initial treatment with anti-PD-1 antibodies (nivolumab/pembrolizumab).

\begin{tabular}{|c|c|c|c|}
\hline & $\begin{array}{c}\text { Nivolumab }(\mathrm{n}=8) \\
\mathrm{n}(\%)\end{array}$ & $\begin{array}{c}\text { Pembrolizumab }(\mathrm{n}=7) \\
\mathrm{n}(\%)\end{array}$ & $\begin{array}{c}\text { Nivolumab+pembrolizumab }(\mathrm{n}=3) \\
\mathrm{n}(\%)\end{array}$ \\
\hline Regimens before anti-PD-1 antibody* & $1.5(1-3)$ & $1.0(2-12)$ & $1.0(1-3)$ \\
\hline Cycles of anti-PD-1 antibody* & $9.5(3-37)$ & $10(2-12)$ & NIV 7.5 (7-32), PEMB $6(1-22)$ \\
\hline \multicolumn{4}{|l|}{ PD-L1 expression } \\
\hline TPS $\geq 50 \%$ & $0(0)$ & $6(85.7)$ & $3(100)$ \\
\hline $1 \% \leq \mathrm{TPS}<50 \%$ & $2(25.0)$ & $1(14.3)$ & $0(0)$ \\
\hline TPS $<1 \%$ & $3(37.5)$ & $0(0)$ & $0(0)$ \\
\hline $\mathrm{NE}^{* *}$ & $3(37.5)$ & $0(0)$ & $0(0)$ \\
\hline PFS, months & $7.7 \pm 6.6$ & $5.6 \pm 4.7$ & NIV $8.4 \pm 7.0$, PEMB $5.0 \pm 5.0$ \\
\hline \multicolumn{4}{|l|}{ Best response } \\
\hline PR & $4(50.0)$ & $3(42.9)$ & $0(0)$ \\
\hline SD & $2(25.0)$ & $1(14.3)$ & NIV 3 (100), PEMB 1 (33.3) \\
\hline PD & $2(25.0)$ & $3(42.9)$ & PEMB 1 (33.3) \\
\hline NE & $0(0)$ & $0(0)$ & PEMB $1(33.3)$ \\
\hline \multicolumn{4}{|l|}{ Treatment prior anti-PD-1 antibody } \\
\hline \multicolumn{4}{|l|}{ Cytotoxic chemotherapy 1} \\
\hline $\mathrm{CBDCA}+$ nabPTX/PTX $\pm \mathrm{BV}$ & $2(25.0)$ & $2(28.6)$ & $2(66.7)$ \\
\hline $\mathrm{CBDCA}+\mathrm{PEM} \pm \mathrm{BV}$ & $4(50.0)$ & $2(28.6)$ & $2(66.7)$ \\
\hline DTX+RAM & $3(37.5)$ & $0(0)$ & $2(66.7)$ \\
\hline Others & $5(62.5)$ & $0(0)$ & $2(66.7)$ \\
\hline
\end{tabular}

*Data shown as median (range). **Data shown as mean \pm standard deviation. ${ }^{1}$ There were overlaps in the number of cycles of cytotoxic chemotherapy. PD-1. Programmed death-1; PD-L1, programmed death ligand 1; TPS, tumor proportion score; NE, not evaluated; PFS, progression-free survival; PR, partial response; SD, stable disease; PD, progressive disease; CBDCA, carboplatin; nabPTX, nanoparticle albumin-bound paclitaxel; PEM, pemetrexed; BV, bevacizumab; DTX, docetaxel; RAM, ramucirumab; NIV, nivolumab; PEMB, pembrolizumab.

study reported that patients who responded well to initial ICI treatment received benefits from ICI retreatment (10). Moreover, the efficacy of retreatment with the same anti-PDL1 antibody has been evaluated in several tumor types and it was found that a limited number of patients had good clinical response (11). These studies all evaluated re-treatment with an anti-PD-1/PD-L1 antibody after original treatment with an
anti-PD-1/PD-L1 antibody. There are no studies evaluating anti-PD-L1 antibody after an anti-PD-1 antibody.

In this study, we evaluated the efficacy and safety of antiPD-L1 (atezolizumab) retreatment after anti-PD-1 (nivolumab/pembrolizumab) treatment. Unfortunately, the results indicated a poor response to atezolizumab after treatment with anti-PD-1 antibodies. One possible 
Table III. Data about patients with subsequent treatment with anti-PDL1 antibody (atezolizumab).

\begin{tabular}{lc}
\hline & $\begin{array}{c}\text { Atezolizumab }(\mathrm{n}=18) \\
\mathrm{n}(\%)\end{array}$ \\
\hline Regimens before atezolizumab* & $3(1-6)$ \\
Cycles of atezolizumab* & $3(2-7)$ \\
PD-L1 expression & \\
TPS $\geq 50 \%$ & $9(50.0)$ \\
$1 \% \leq$ TPS $<50 \%$ & $3(16.7)$ \\
TPS $<1 \%$ & $3(16.7)$ \\
NE** & $3(16.7)$ \\
PFS, month & $2.9 \pm 1.8$ \\
Best response & \\
PR & $0(0)$ \\
SD & $7(38.9)$ \\
PD & $11(61.1)$ \\
NE & $0(0)$ \\
Disease control rate, $\%$ & 38.9 \\
Number of patients receiving treatment & \\
between anti-PD-1 antibody and atezolizumab & $9(50.0)$ \\
Types of treatment between anti-PD-1 antibody & \\
and atezolizumab & \\
Cytotoxic chemotherapy ${ }^{*}$ & \\
CBDCA+nabPTX/PTX $\pm B V$ & \\
CBDCA+PEM \pm BV & $2(11.1)$ \\
DTX+RAM & $1(5.6)$ \\
Others & $3(16.7)$ \\
\hline
\end{tabular}

*Data shown as median (range). **Data shown as mean \pm standard deviation. ${ }^{1}$ There were overlaps in the number of cycles of cytotoxic chemotherapy. PD-1, Programmed death-1; PD-L1, programmed death ligand 1; TPS, tumor proportion score; NE, not evaluated; PFS, progression-free survival; $\mathrm{PR}$, partial response; $\mathrm{SD}$, stable disease; $\mathrm{PD}$, progressive disease; CBDCA, carboplatin; nabPTX, nanoparticle albumin-bound paclitaxel; PEM, pemetrexed; BV, bevacizumab; DTX, docetaxel; RAM, ramucirumab.

explanation for this outcome is that a large number of our patients received atezolizumab as a third- or later-line regimen, many patients had experienced multiple anti-cancer treatments, including cytotoxic chemotherapy and immunotherapy. Their physically exhausted status might cause the depressed immune response that was observed compared to those of previous clinical trials. Furthermore, half of the patients in our study had a poor performance status ( $\geq 2$ ) at the time of treatment with atezolizumab, which could also be related to the exhausted status.

Atezolizumab also has different mechanisms of action than anti-PD-1 antibodies. Whereas anti-PD-1 antibodies block the binding of PD-1 to both PD-L1 and PD-L2, anti-PD-L1 antibodies block the interaction of PD-1 and PD-L1 but do not block the binding of PD-1 to PD-L2 (12). Furthermore, antiPD-L1 antibodies block the interaction of tumor cells and B7.1expressing cells, which suppress T-cell activation and cytokine production (12). Based on these differences several hypotheses
Table IV. Profiles of immune-related adverse events.

\begin{tabular}{lccccc}
\hline & $\begin{array}{c}\text { Initial anti-PD-1 } \\
\text { antibody }\end{array}$ & & \multicolumn{2}{c}{$\begin{array}{c}\text { Subsequent } \\
\text { atezolizumab }\end{array}$} \\
\cline { 2 - 3 } \cline { 5 - 6 } & $\mathrm{G} 1$ & $\geq \mathrm{G} 2$ & & $\mathrm{G} 1$ & $\geq \mathrm{G} 2$ \\
\hline Rash & 1 & 2 & & 1 & 6 \\
Infection & 0 & 3 & & 0 & 4 \\
Elevation of liver enzyme & 1 & 0 & & 1 & 0 \\
Fatigue & 1 & 0 & & 2 & 0 \\
Interstitial pneumonia & 1 & 2 & & 0 & 1 \\
Diarrhea & 1 & 1 & & 0 & 0 \\
Fever & 2 & 0 & & 4 & 1 \\
mental disorder & 0 & 0 & & 0 & 2 \\
Hypothyroidism & 0 & 1 & & 0 & 1 \\
\hline
\end{tabular}

Data are shown as number of patients experienced each adverse event. PD-1, Programmed death-1; G, grade according to the Common Terminology Criteria for Adverse Events version 4.0.

could be suggested. Patients with high tumor PD-L2 expression might have had a poor response. Induced expression of PD-L2 after blocking PD-L1 (following treatment with anti-PD-1 antibodies) might attenuate the efficacy of anti-PD-L1 antibody. In preclinical experiments, although inhibiting PD-L2 alone was suggested to have no anti-tumor effects, immune inhibitory effects of PD-L2 were shown when the PD-1/PD-L1 interaction was attenuated (13). Furthermore, a recent clinical study showed that high PD-L2 expression was related to poor prognosis (14). These data might partially explain our results.

Regarding the AEs in this study, skin rash and fever were more frequently observed during treatment with atezolizumab, but they were not severe. Severe infection was observed after both initial anti-PD-1 and subsequent atezolizumab treatments. Another recent study also indicated the development of infectious diseases in patients treated with ICIs (15). Taken together, our results suggest that particular attention should be paid to these potential side-effects in patients receiving ICIs.

There were several limitations in our study. Our study was retrospective, and the setting was a single institution, which allowed us only a limited number of patients. The timing of treatment as well as regimens were chosen by the attending doctors and therefore not standardized between patients. Statistical analysis could not be performed due to the small sample size, thus the results of this study are preliminary. Furthermore, we did not evaluate the expression of PD-L2 or B7.1. We, therefore, did not assess the relationship between PD-L2 expression, B7.1 expression, and clinical outcome.

In conclusion, patients previously treated with anti PD-1 antibodies received only limited benefit from subsequent administration of atezolizumab in this study. The results of ongoing clinical trials evaluating retreatment with anti-PD- 
L1 antibody after anti-PD-1 antibody treatment will provide a clearer overall picture.

\section{Conflicts of Interest}

The Authors have no conflicts of interest to declare.

\section{Authors' Contributions}

KF designed this study. KF, NU, YY, OK, MO, KN, SS and TM recruited and cared for the patients. KF drafted and revised the manuscript. NK and TM supervised this study and revised the manuscript.

\section{Acknowledgements}

The thank Miki Koda for her assistance with data collection.

\section{References}

1 Brahmer J, Reckamp KL, Baas P, Crino L, Eberhardt WE, Poddubskaya E, Antonia S, Pluzanski A, Vokes EE, Holgado E, Waterhouse D, Ready N, Gainor J, Aren Frontera O, Havel L, Steins M, Garassino MC, Aerts JG, Domine M, Paz-Ares L, Reck M, Baudelet C, Harbison CT, Lestini B and Spigel DR: Nivolumab versus Docetaxel in advanced squamous-cell nonsmall-cell lung cancer. N Engl J Med 373: 123-135, 2015. PMID: 26412456. DOI: 10.1056/NEJMoa1507643

2 Borghaei H, Paz-Ares L, Horn L, Spigel DR, Steins M, Ready NE, Chow LQ, Vokes EE, Felip E, Holgado E, Barlesi F, Kohlhaufl M, Arrieta O, Burgio MA, Fayette J, Lena H, Poddubskaya E, Gerber DE, Gettinger SN, Rudin CM, Rizvi N, Crino L, Blumenschein GR, Antonia SJ, Dorange C, Harbison CT, Graf Finckenstein F and Brahmer JR: Nivolumab versus Docetaxel in advanced nonsquamous non-small-cell lung cancer. N Engl J Med 373: 1627-1639, 2015. PMID: 26412456. DOI: 10.1056/NEJMoa1507643

3 Herbst RS, Baas P, Kim DW, Felip E, Perez-Gracia JL, Han JY, Molina J, Kim JH, Arvis CD, Ahn MJ, Majem M, Fidler MJ, de Castro G, Garrido M, Lubiniecki GM, Shentu Y, Im E, DolledFilhart M and Garon EB: Pembrolizumab versus docetaxel for previously treated, PD-L1-positive, advanced non-small-cell lung cancer (KEYNOTE-010): A randomised controlled trial. Lancet 387: 1540-1550, 2016. PMID: 26712084. DOI: 10.1016/ S0140-6736(15)01281-7

4 Rittmeyer A, Barlesi F, Waterkamp D, Park K, Ciardiello F, von Pawel J, Gadgeel SM, Hida T, Kowalski DM, Dols MC, Cortinovis DL, Leach J, Polikoff J, Barrios C, Kabbinavar F, Frontera OA, De Marinis F, Turna H, Lee JS, Ballinger M, Kowanetz M, He P, Chen DS, Sandler A and Gandara DR; OAK Study Group: Atezolizumab versus docetaxel in patients with previously treated non-small-cell lung cancer (OAK): a phase 3, open-label, multicentre randomised controlled trial. Lancet 389: 255-265, 2017. PMID: 27979383. DOI: 10.1016/S0140-6736(16)32517-X

5 Antonia SJ, Villegas A, Daniel D, Vicente D, Murakami S, Hui R, Kurata T, Chiappori A, Lee KH, de Wit M, Cho BC, Bourhaba M, Quantin X, Tokito T, Mekhail T, Planchard D, Kim YC, Karapetis CS, Hiret S, Ostoros G, Kubota K, Gray JE, PazAres L, de Castro Carpeno J, Faivre-Finn C, Reck M,
Vansteenkiste J, Spigel DR, Wadsworth C, Melillo G, Taboada M, Dennis PA and Ozguroglu M; PACIFIC Investigators: Overall survival with durvalumab after chemoradiotherapy in stage III NSCLC. N Engl J Med 379: 2342-2350, 2018. PMID: 30280658. DOI: 10.1056/NEJMoa1809797

6 The Japan Lung Cancer Society. Guidelines for Diagnosis and Treatment of the Lung Cancer 2018 [In Japanese]. Available from: https://www.haigan.gr.jp/modules/guideline/index.php? content_id=3

7 Blasig H, Bender C, Hassel JC, Eigentler TK, Sachse MM, Hiernickel J, Koop A, Satzger I and Gutzmer R: Reinduction of PD1-inhibitor therapy: First experience in eight patients with metastatic melanoma. Melanoma Res 27: 321-325, 2017. PMID: 28257394. DOI: 10.1097/CMR.0000000000000341

8 Nomura M, Otsuka A, Kondo T, Nagai H, Nonomura Y, Kaku Y, Matsumoto $\mathrm{S}$ and Muto M: Efficacy and safety of retreatment with nivolumab in metastatic melanoma patients previously treated with nivolumab. Cancer Chemother Pharmacol 80: 999-1004, 2017. PMID: 28983657. DOI: 10.1007/s00280-017-3444-0

9 Fujita K, Uchida N, Kanai O, Okamura M, Nakatani K and Mio $\mathrm{T}$ : Retreatment with pembrolizumab in advanced non-small cell lung cancer patients previously treated with nivolumab: emerging reports of 12 cases. Cancer Chemother Pharmacol 81: 1105-1109, 2018. PMID: 29675747. DOI: 10.1007/s00280-018-3585-9

10 Niki M, Nakaya A, Kurata T, Yoshioka H, Kaneda T, Kibata K, Ogata $\mathrm{M}$ and Nomura S: Immune checkpoint inhibitor rechallenge in patients with advanced non-small cell lung cancer. Oncotarget 9: 32298-32304, 2018. PMID: 30190787. DOI: 10.18632/oncotarget.25949

11 Bernard-Tessier A, Baldini C, Martin P, Champiat S, Hollebecque A, Postel-Vinay S, Varga A, Bahleda R, Gazzah A, Michot JM, Ribrag V, Armand JP, Marabelle A, Soria JC and Massard C: Outcomes of long-term responders to anti-programmed death 1 and anti-programmed death ligand 1 when being rechallenged with the same anti-programmed death 1 and anti-programmed death ligand 1 at progression. Eur J Cancer 101: 160-164, 2018. PMID: 30071444. DOI: 10.1016/ j.ejca.2018.06.005

12 Mahoney KM, Rennert PD and Freeman GJ: Combination cancer immunotherapy and new immunomodulatory targets. Nat Rev Drug Discov 14: 561-584, 2015. PMID: 26228759. DOI: $10.1038 /$ nrd4591

13 Umezu D, Okada N, Sakoda Y, Adachi K, Ojima T, Yamaue H, Eto $\mathrm{M}$ and Tamada K: Inhibitory functions of PD-L1 and PDL2 in the regulation of anti-tumor immunity in murine tumor microenvironment. Cancer Immunol Immunother 68: 201-211, 2019. PMID: 30357491. DOI: 10.1007/s00262-018-2263-4

14 Takamori S, Takada K, Azuma K, Jogo T, Shimokawa M, Toyokawa G, Hirai F, Tagawa T, Kawahara A, Akiba J, Okamoto I, Nakanishi Y, Oda Y, Hoshino T and Maehara Y: Prognostic impact of programmed death-ligand 2 expression in primary lung adenocarcinoma patients. Ann Surg Oncol, 2019. PMID: 30815801. DOI: 10.1245/s10434-019-07231-z

15 Fujita K, Kim YH, Kanai O, Yoshida H, Mio T and Hirai T: Emerging concerns of infectious diseases in lung cancer patients receiving immune checkpoint inhibitor therapy. Respir Med 146: 66-70, 2019. PMID: 30665520. DOI: 10.1016/j.rmed.2018. 11.021

Received March 12, 2019 Revised April 18, 2019 Accepted April 19, 2019 